\title{
The Method of Lyapunov Function and Exponential Stability of Impulsive Delay Systems with Delayed Impulses
}

\author{
Pei Cheng, ${ }^{1}$ Feiqi Deng, ${ }^{2}$ and Lianglong Wang ${ }^{1}$ \\ ${ }^{1}$ School of Mathematical Sciences, Anhui University, Hefei 230601, China \\ ${ }^{2}$ Systems Engineering Institute, South China University of Technology, Guangzhou 510640, China \\ Correspondence should be addressed to Pei Cheng; chengpei_pi@163.com
}

Received 4 September 2013; Revised 5 November 2013; Accepted 5 November 2013

Academic Editor: Bo Shen

Copyright (c) 2013 Pei Cheng et al. This is an open access article distributed under the Creative Commons Attribution License, which permits unrestricted use, distribution, and reproduction in any medium, provided the original work is properly cited.

\begin{abstract}
This paper investigates the exponential stability of general impulsive delay systems with delayed impulses. By using the Lyapunov function method, some Lyapunov-based sufficient conditions for exponential stability are derived, which are more convenient to be applied than those Razumikhin-type conditions in the literature. Their applications to linear impulsive systems with time-varying delays are also proposed, and a set of sufficient conditions for exponential stability is provided in terms of matrix inequalities. Meanwhile, two examples are discussed to illustrate the effectiveness and advantages of the results obtained.
\end{abstract}

\section{Introduction}

Impulsive dynamical systems have received considerable attention during the recent decades since they provide a natural framework for mathematical modeling of many realworld evolutionary processes where the states undergo abrupt changes at certain instants (see, e.g., [1-4]). On the other hand, time delays often appear in practical systems and may poorly affect the performance of a system. Stability and stabilization of such systems are of both theoretical and practical importance. Therefore, the study of time-delay systems has attracted great attention over the past few years (see, e.g., [5-10]). An area of particular interest has been the stability analysis and stabilization of impulsive delay systems (IDSs), and there is extensive literature on this field (see, e.g., [1126]). For instance, in [11-14], the robust stability for uncertain impulsive system with time-delay was studied, and the linear matrix inequalities approach to the stability was presented. In [15-26], the Lyapunov function or Lyapunov functional coupled with the Razumikhin techniques was suggested for the exponential stability and asymptotical stability of IDSs.

In the previous works on stability and stabilization of IDSs, the impulses are assumed to take the form of $\Delta x\left(t_{k}\right)=I_{k}\left(t_{k}, x\left(t_{k}^{-}\right)\right)$, which indicates that the state "jump" at impulse times $t_{k}$ is only related to the present state variables (see, e.g., [18-26]). But, in most cases, it is more applicable that the state variables on the impulses are also related to the past states. For example, in the transmission of the impulse information, input delays are often encountered. So, compared with the nondelayed impulses described above, it is much more meaningful to model the impulses as

$$
\Delta x\left(t_{k}\right)=I_{k}\left(t_{k}, x\left(t_{k}^{-}\right)\right)+J_{k}\left(t_{k}, x_{t_{k}^{-}}\right) .
$$

In fact, there have been several attempts in the literature to study the stability and control problems of a particular class of delayed impulsive systems [27, 28]. For example, Lian et al. [27] investigated the optimal control problem of linear continuous-time systems possessing delayed discretetime controllers in networked control systems. For nonlinear impulsive systems, Khadra et al. [28] studied the impulsive synchronization problem coupled by linear delayed impulses. By using the Razumikhin techniques, some sufficient conditions for asymptotic stability and exponential stability of general IDS-DI were established in [29-32], and sufficient conditions for exponential stability of impulsive stochastic functional differential systems with delayed impulses were obtained in [33]. 
In this paper, we will further investigate the stability of IDS-DI. By using the Lyapunov functions, some sufficient conditions ensuring exponential stability of IDS-DI are derived, which are more convenient to be applied than those Razumikhin-type conditions in [31,32]. Their applications to linear impulsive systems with time-varying delays are also proposed, and a set of sufficient conditions for exponential stability are derived in terms of matrix inequalities.

\section{Preliminaries}

Let $\mathbb{R}$ denote the set of real numbers, $\mathbb{R}_{+}$the set of nonnegative real numbers, $\mathbb{Z}_{+}$the set of positive integers, and $\mathbb{R}^{n}$ the $n$-dimensional real space equipped with the Euclidean norm $|\cdot|$. Let $\tau>0$, and let $P C\left([-\tau, 0] ; \mathbb{R}^{n}\right)=\left\{\varphi:[-\tau, 0] \rightarrow \mathbb{R}^{n} \mid\right.$ $\varphi\left(t^{+}\right)=\varphi(t)$ for all $t \in[-\tau, 0), \varphi\left(t^{-}\right)$exists and $\varphi\left(t^{-}\right)=\varphi(t)$ for all but at most a finite number of points $t \in(-\tau, 0]\}$ be with the norm $\|\varphi\|=\sup _{-\tau \leqslant \theta \leqslant 0}|\varphi(\theta)|$, where $\varphi\left(t^{+}\right)$and $\varphi\left(t^{-}\right)$ denote the right-hand and left-hand limits of function $\varphi(t)$ at $t$ respectively.

Consider the IDS-DI described by the state equations

$$
\begin{gathered}
\dot{x}(t)=f\left(t, x_{t}\right), \quad t \neq t_{k}, t \geqslant t_{0}, \\
\Delta x\left(t_{k}\right)=I_{k}\left(t_{k}, x\left(t_{k}^{-}\right)\right)+J_{k}\left(t_{k}, x_{t_{k}^{-}}\right), \quad k \in \mathbb{Z}_{+}, \\
x_{t_{0}}=\phi(s), \quad s \in[-\tau, 0],
\end{gathered}
$$

where $x \in \mathbb{R}^{n}, f: \mathbb{R}_{+} \times \mathbb{C} \rightarrow \mathbb{R}^{n}, I_{k}: \mathbb{R}_{+} \times \mathbb{R}^{n} \rightarrow \mathbb{R}^{n}, J_{k}$ : $\mathbb{R}_{+} \times \mathbb{C} \rightarrow \mathbb{R}^{n}, \phi \in P C\left([-\tau, 0] ; \mathbb{R}^{n}\right)$, and $\mathbb{C}$ is an open set in $\operatorname{PC}\left([-\tau, 0] ; \mathbb{R}^{n}\right)$. The fixed moments of impulse times $\left\{t_{k}, k \in\right.$ $\mathbb{Z}_{+}$\} satisfy $0 \leqslant t_{0}<t_{1}<\cdots<t_{k}<\cdots$ and $t_{k} \rightarrow \infty$ (as $k \rightarrow \infty)$ and $\Delta x\left(t_{k}\right)=x\left(t_{k}\right)-x\left(t_{k}^{-}\right) ; x_{t}, x_{t^{-}} \in P C\left([-\tau, 0] ; \mathbb{R}^{n}\right)$ are defined as $x_{t}=x(t+\theta)$ and $x_{t^{-}}=x\left(t^{-}+\theta\right)$ for $\theta \in[-\tau, 0]$ respectively.

Throughout this paper, we assume that $f, I_{k}$, and $J_{k}, k \in$ $\mathbb{Z}_{+}$, satisfy the necessary conditions for the global existence and uniqueness of solutions for all $t \geqslant t_{0}$ (refer to $\left.[3,34,35]\right)$. Then, for any $\phi \in P C\left([-\tau, 0] ; \mathbb{R}^{n}\right)$, there exists a unique function satisfying system (2) denoted by $x\left(t ; t_{0}, \phi\right)$, which is continuous on the right-hand side and limitable on the lefthand side. Moreover, we assume that $f(t, 0) \equiv 0, I_{k}\left(t_{k}, 0\right) \equiv 0$, and $J_{k}\left(t_{k}, 0\right) \equiv 0, k \in \mathbb{Z}_{+}$, which implies that $x(t) \equiv 0$ is a solution of (2), which is called the trivial solution.

At the end of this section, let us introduce the following definitions.

Definition 1. A function $V:\left[t_{0}-\tau, \infty\right) \times \mathbb{R}^{n} \rightarrow \mathbb{R}_{+}$belongs to class $v_{0}$ if the following are satisfied.

(i) $V$ is continuous on each of the sets $\left[t_{k-1}, t_{k}\right) \times \mathbb{R}^{n}$, and for each $x, y \in \mathbb{R}^{n}, t \in\left[t_{k-1}, t_{k}\right), k \in \mathbb{Z}_{+}$, $\lim _{(t, y) \rightarrow\left(t_{k}^{-}, x\right)} V(t, y)=V\left(t_{k}^{-}, x\right)$ exists.

(ii) $V(t, x)$ is locally Lipschitz in $x \in \mathbb{R}^{n}$, and $V(t, 0) \equiv 0$ for all $t \geqslant t_{0}$.
Definition 2. Given a function $V \in v_{0}$, the upper right-hand Dini derivative of $V$ with respect to system (2) is defined by

$$
\begin{aligned}
& D^{+} V(t, \psi(0)) \\
& \quad=\limsup _{h \rightarrow 0^{+}} \frac{1}{h}[V(t+h, \psi(0)+h f(t, \psi))-V(t, \psi(0))]
\end{aligned}
$$

for $(t, \psi) \in\left[t_{0}, \infty\right) \times P C\left([-\tau, 0] ; \mathbb{R}^{n}\right)$.

Definition 3. The trivial solution of system (2) or, simply, system (2) is said to be exponentially stable if there is a pair of positive constants $\lambda, C$ such that, for any initial data $x_{t_{0}}=$ $\phi \in P C\left([-\tau, 0] ; \mathbb{R}^{n}\right)$, the solution $x\left(t ; t_{0}, \phi\right)$ satisfies

$$
\left|x\left(t ; t_{0}, \phi\right)\right| \leqslant C\|\phi\| e^{-\lambda\left(t-t_{0}\right)}, \quad t \geqslant t_{0} .
$$

\section{Main Results}

In this section, we will analyze the exponential stability of system (2) by employing the Lyapunov functions.

Theorem 4. Assume that $V \in v_{0}$ and there exist constants $p>$ $0, c_{1}>0, c_{2}>0, \eta_{2} \geqslant 0, d_{1 k}>0, d_{2 k} \geqslant 0, k \in \mathbb{Z}_{+}, \eta_{1}$, and $\delta$ such that

(i) $c_{1}|x|^{p} \leqslant V(t, x) \leqslant c_{2}|x|^{p}$ for all $(t, x) \in\left[t_{0}-\tau, \infty\right) \times \mathbb{R}^{n}$;

(ii) $V\left(t_{k}, \varphi(0)+I_{k}\left(t_{k}, \varphi(0)\right)+J_{k}\left(t_{k}, \varphi\right)\right) \leqslant d_{1 k} V\left(t_{k}^{-}, \varphi(0)\right)+$ $d_{2 k} \sup _{\theta \in[-\tau, 0]} V\left(t_{k}^{-}+\theta, \varphi(\theta)\right)$ for all $\varphi \in P C([-\tau, 0]$; $\left.\mathbb{R}^{n}\right), k \in \mathbb{Z}_{+}$;

(iii) $D^{+} V(t, \varphi) \leqslant \eta_{1} V(t, \varphi(0))+\eta_{2} \sup _{\theta \in[-\tau, 0]} V(t+\theta, \varphi(\theta))$ for all $\varphi \in P C\left([-\tau, 0] ; \mathbb{R}^{n}\right)$ and $t \geqslant t_{0}, t \neq t_{k}, k \in \mathbb{Z}_{+} ;$

(iv) $\ln \left(d_{1 k}+d_{2 k} \max _{\theta \in[-\tau, 0]} e^{\eta_{1} \theta}\right) \leqslant \delta\left(t_{k}-t_{k-1}\right)$ for each $k \in$ $\mathbb{Z}_{+} ;$

(v) $\delta+\eta_{1}+\gamma \eta_{2}<0$, where $\gamma=\sup _{k \in \mathbb{Z}_{+}}\left\{e^{\delta\left(t_{k}-t_{k-1}\right)}\right.$, $\left.1 / e^{\delta\left(t_{k}-t_{k-1}\right)}\right\}$.

Then, system (2) is exponentially stable, and the convergence rate should not be greater than $\lambda / p$, where $\lambda$ is the unique positive solution of $\lambda+\delta+\eta_{1}+\gamma \eta_{2} e^{\lambda \tau}=0$.

Proof. Fix any initial data $\phi \in P C\left([-\tau, 0] ; \mathbb{R}^{n}\right)$, and write $x\left(t ; t_{0}, \phi\right)=x(t)$ and $V(t, x(t))=V(t)$ simply. Set $W(t)=$ $e^{-\eta_{1}\left(t-t_{0}\right)} V(t), t \in\left[t_{0}-\tau, \infty\right)$. For each $k \in \mathbb{Z}_{+}$, by condition (ii), we have

$$
\begin{aligned}
W\left(t_{k}\right)= & e^{-\eta_{1}\left(t_{k}-t_{0}\right)} V\left(t_{k}\right) \\
\leqslant & e^{-\eta_{1}\left(t_{k}-t_{0}\right)}\left[d_{1 k} V\left(t_{k}^{-}\right)+d_{2 k} \sup _{\theta \in[-\tau, 0]} V\left(t_{k}^{-}+\theta\right)\right] \\
\leqslant & d_{1 k} e^{-\eta_{1}\left(t_{k}-t_{0}\right)} V\left(t_{k}^{-}\right) \\
& +\alpha d_{2 k} \sup _{\theta \in[-\tau, 0]}\left(V\left(t_{k}^{-}+\theta\right) e^{-\eta_{1}\left(t_{k}+\theta-t_{0}\right)}\right) \\
= & d_{1 k} W\left(t_{k}^{-}\right)+\alpha d_{2 k} \sup _{\theta \in[-\tau, 0]} W\left(t_{k}^{-}+\theta\right)
\end{aligned}
$$

where $\alpha=\max _{\theta \in[-\tau, 0]} e^{\eta_{1} \theta}$. 
On the other hand, by condition (iii), we know that, for any $t \neq t_{k}, k \in \mathbb{Z}_{+}$,

$$
\begin{aligned}
D^{+} W(t) & =e^{-\eta_{1}\left(t-t_{0}\right)}\left[-\eta_{1} V(t)+D^{+} V(t)\right] \\
& \leqslant \eta_{2} e^{-\eta_{1}\left(t-t_{0}\right)} \sup _{\theta \in[-\tau, 0]} V(t+\theta) .
\end{aligned}
$$

For $t \in\left[t_{0}, t_{1}\right)$, integrating inequality (6) from $t_{0}$ to $t$, we obtain

$$
W(t) \leqslant W\left(t_{0}\right)+\int_{t_{0}}^{t} \eta_{2} e^{-\eta_{1}\left(s-t_{0}\right)} \sup _{\theta \in[-\tau, 0]} V(s+\theta) d s .
$$

This implies that

$$
W\left(t_{1}^{-}\right) \leqslant W\left(t_{0}\right)+\int_{t_{0}}^{t_{1}} \eta_{2} e^{-\eta_{1}\left(s-t_{0}\right)} \sup _{\theta \in[-\tau, 0]} V(s+\theta) d s .
$$

For $t \in\left[t_{1}, t_{2}\right)$, by the same method, together with (5) and (8), we have

$$
\begin{aligned}
& W(t) \leqslant W\left(t_{1}\right)+\int_{t_{1}}^{t} \eta_{2} e^{-\eta_{1}\left(s-t_{0}\right)} \sup _{\theta \in[-\tau, 0]} V(s+\theta) d s \\
& \leqslant d_{11} W\left(t_{1}^{-}\right)+\alpha d_{21} \sup _{\theta \in[-\tau, 0]} W\left(t_{1}^{-}+\theta\right) \\
& +\int_{t_{1}}^{t} \eta_{2} e^{-\eta_{1}\left(s-t_{0}\right)} \sup _{\theta \in[-\tau, 0]} V(s+\theta) d s \\
& \leqslant d_{11}\left[W\left(t_{0}\right)+\int_{t_{0}}^{t_{1}} \eta_{2} e^{-\eta_{1}\left(s-t_{0}\right)} \sup _{\theta \in[-\tau, 0]} V(s+\theta) d s\right] \\
& +\alpha d_{21}\left[W\left(t_{0}\right)+\sup _{\theta \in[-\tau, 0]} \int_{t_{0}}^{t_{1}+\theta} \eta_{2} e^{-\eta_{1}\left(s-t_{0}\right)}\right. \\
& \left.\times \sup _{\theta \in[-\tau, 0]} V(s+\theta) d s\right] \\
& +\int_{t_{1}}^{t} \eta_{2} e^{-\eta_{1}\left(s-t_{0}\right)} \sup _{\theta \in[-\tau, 0]} V(s+\theta) d s \\
& \leqslant\left(d_{11}+\alpha d_{21}\right) W\left(t_{0}\right) \\
& +\left(d_{11}+\alpha d_{21}\right) \int_{t_{0}}^{t_{1}} \eta_{2} e^{-\eta_{1}\left(s-t_{0}\right)} \sup _{\theta \in[-\tau, 0]} V(s+\theta) d s \\
& +\int_{t_{1}}^{t} \eta_{2} e^{-\eta_{1}\left(s-t_{0}\right)} \sup _{\theta \in[-\tau, 0]} V(s+\theta) d s .
\end{aligned}
$$

By induction, we have, for $t \in\left[t_{k-1}, t_{k}\right), k \in \mathbb{Z}_{+}$,

$$
\begin{aligned}
W(t) \leqslant & W\left(t_{0}\right) \prod_{t_{0}<t_{i} \leqslant t}\left(d_{1 i}+\alpha d_{2 i}\right) \\
& +\int_{t_{0}}^{t} \prod_{s<t_{i} \leqslant t}\left(d_{1 i}+\alpha d_{2 i}\right) \eta_{2} e^{-\eta_{1}\left(s-t_{0}\right)} \sup _{\theta \in[-\tau, 0]} V(s+\theta) d s .
\end{aligned}
$$

Thus, for $t>t_{0}$, we get

$$
\begin{aligned}
V(t) \leqslant & V\left(t_{0}\right) e^{\eta_{1}\left(t-t_{0}\right)} \prod_{t_{0}<t_{i} \leqslant t}\left(d_{1 i}+\alpha d_{2 i}\right) \\
& +\int_{t_{0}}^{t} \prod_{s<t_{i} \leqslant t}\left(d_{1 i}+\alpha d_{2 i}\right) \eta_{2} e^{\eta_{1}(t-s)} \sup _{\theta \in[-\tau, 0]} V(s+\theta) d s .
\end{aligned}
$$

Let $t_{j_{1}}, t_{j_{2}}, \ldots, t_{j_{m}}$ be impulse points in $(s, t], t>s$. In view of condition (iv), we get

$$
\begin{aligned}
\prod_{s<t_{i} \leqslant t} & \left(d_{1 i}+\alpha d_{2 i}\right) \\
& =\left(d_{1 j_{1}}+\alpha d_{2 j_{1}}\right)\left(d_{1 j_{2}}+\alpha d_{2 j_{2}}\right) \cdots\left(d_{1 j_{m}}+\alpha d_{2 j_{m}}\right) \\
& \leqslant e^{\delta\left(t_{j_{1}}-t_{j_{1}-1}\right)} e^{\delta\left(t_{j_{2}}-t_{j_{1}}\right)} \cdots e^{\delta\left(t_{j_{m}}-t_{j_{m-1}}\right)}=e^{\delta\left(t_{j_{m}}-t_{j_{1}-1}\right)} \\
& =e^{\delta(t-s)} e^{\delta\left(t_{j_{m}}-t\right)} e^{\delta\left(s-t_{j_{l}-1}\right)} \leqslant \gamma e^{\delta(t-s)},
\end{aligned}
$$

where $t_{j_{1}-1}$ is the first impulsive point before $t_{j_{1}}$ and satisfies $t_{j_{1}-1}<s$. Submitting this into inequality (11), then, for $t>t_{0}$,

$$
\begin{aligned}
V(t) \leqslant & \gamma e^{\left(\eta_{1}+\delta\right)\left(t-t_{0}\right)} V\left(t_{0}\right) \\
& +\int_{t_{0}}^{t} \gamma \eta_{2} e^{\left(\eta_{1}+\delta\right)(t-s)} \sup _{\theta \in[-\tau, 0]} V(s+\theta) d s .
\end{aligned}
$$

Let $\Phi(\lambda)=\lambda+\eta_{1}+\delta+\gamma \eta_{2} e^{\lambda \tau}$. Then, condition (v) implies $\Phi(0)<0$. Moreover, $\Phi(+\infty)=+\infty$, and $\Phi^{\prime}(\lambda)=1+$ $\tau \gamma \eta_{2} e^{\lambda \tau}>0$. Hence, $\Phi(\lambda)=0$ has a unique positive solution $\lambda$. Next, we claim that

$$
V(t) \leqslant \gamma \sup _{\theta \in[-\tau, 0]} V\left(t_{0}+\theta\right) e^{-\lambda\left(t-t_{0}\right)}, \quad t \geqslant t_{0}-\tau
$$

Obviously,

$$
\begin{array}{r}
V(t) \leqslant \sup _{\theta \in[-\tau, 0]} V\left(t_{0}+\theta\right) \leqslant \gamma \sup _{\theta \in[-\tau, 0]} V\left(t_{0}+\theta\right) e^{-\lambda\left(t-t_{0}\right)}, \\
t \in\left[t_{0}-\tau, t_{0}\right] .
\end{array}
$$

So we only need to prove (14) for $t>t_{0}$. Suppose not; then there exists a $t^{*} \in\left(t_{0},+\infty\right)$ such that

$$
\begin{gathered}
V\left(t^{*}\right)>\gamma \sup _{\theta \in[-\tau, 0]} V\left(t_{0}+\theta\right) e^{-\lambda\left(t^{*}-t_{0}\right)}, \\
V(t) \leqslant \gamma \sup _{\theta \in[-\tau, 0]} V\left(t_{0}+\theta\right) e^{-\lambda\left(t-t_{0}\right)}, \quad t \in\left[t_{0}-\tau, t^{*}\right) .
\end{gathered}
$$


Thus, from (13), (17), and $\Phi(\lambda)=0$, we see that

$$
\begin{aligned}
V\left(t^{*}\right) \leqslant & \gamma \sup _{\theta \in[-\tau, 0]} V\left(t_{0}+\theta\right) e^{\left(\eta_{1}+\delta\right)\left(t^{*}-t_{0}\right)} \\
& +\gamma \int_{t_{0}}^{t^{*}} \eta_{2} e^{\left(\eta_{1}+\delta\right)\left(t^{*}-s\right)} \sup _{\theta \in[-\tau, 0]} V(s+\theta) d s \\
\leqslant & \gamma \sup _{\theta \in[-\tau, 0]} V\left(t_{0}+\theta\right) e^{\left(\eta_{1}+\delta\right)\left(t^{*}-t_{0}\right)} \\
& +\gamma \int_{t_{0}}^{t^{*}} \gamma \eta_{2} e^{\lambda \tau} e^{\left(\eta_{1}+\delta\right)\left(t^{*}-s\right)} e^{-\lambda\left(s-t_{0}\right)} \sup _{\theta \in[-\tau, 0]} V\left(t_{0}+\theta\right) d s \\
= & \gamma \sup _{\theta \in[-\tau, 0]} V\left(t_{0}+\theta\right) e^{-\lambda\left(t^{*}-t_{0}\right)},
\end{aligned}
$$

which is a contradiction. Therefore, (14) holds.

Then, it follows from (14) and condition (i) that

$$
\begin{aligned}
c_{1}|x(t)|^{p} & \leqslant V(t) \leqslant \gamma \sup _{\theta \in[-\tau, 0]} V\left(t_{0}+\theta\right) e^{-\lambda\left(t-t_{0}\right)} \\
& \leqslant \gamma c_{2}\|\phi\|^{p} e^{-\lambda\left(t-t_{0}\right)}, \quad t \geqslant t_{0},
\end{aligned}
$$

which implies that

$$
|x(t)| \leqslant C\|\phi\| e^{(-\lambda / p)\left(t-t_{0}\right)}, \quad t \geqslant t_{0},
$$

where $C=\left(\gamma c_{2} / c_{1}\right)^{1 / p}$. This completes the proof.

Remark 5. The parameters $d_{1 k}$ and $d_{2 k}$ in condition (ii) describe the influence of impulses on the stability of the underlying continuous systems. Conditions (iv) and (v) in Theorem 4 show that the system will be stable if the impulses frequency and amplitude are suitably related to the increase or decrease of the continuous flows.

Remark 6. It is well known that the Razumikhin techniques are very effective in the study of stability problems for ordinary and functional differential systems. However, when we use the Razumikhin techniques, we need to choose an appropriate minimal class of functionals relative to which the derivative of the Lyapunov function or Lyapunov functional is estimated, which is not entirely convenient. In this sense, Theorem 4 is more convenient to be applied than those Razumikhin-type theorems in [31, 32].

Let $J_{k} \equiv 0$ in system (2); then we have the following IDS (see, e.g., [19-24]):

$$
\begin{gathered}
\dot{x}(t)=f\left(x_{t}, t\right), \quad t \neq t_{k}, t \geqslant t_{0}, \\
\Delta x\left(t_{k}\right)=I_{k}\left(x\left(t_{k}^{-}\right), t_{k}\right), \quad k \in \mathbb{Z}_{+}, \\
x_{t_{0}}=\phi(s), \quad s \in[-\tau, 0] .
\end{gathered}
$$

For system (21), we have the following results by Theorem 4 .

Theorem 7. Assume that $V \in v_{0}$ and there exist constants $p>$ $0, c_{1}>0, c_{2}>0, \eta_{2} \geqslant 0, d_{k}>0, k \in \mathbb{Z}_{+}, \eta_{1}$, and $\delta$ such that (i) $c_{1}|x|^{p} \leqslant V(t, x) \leqslant c_{2}|x|^{p}$ for all $(t, x) \in\left[t_{0}-\tau, \infty\right) \times \mathbb{R}^{n}$;

(ii) $V\left(t_{k}, \varphi(0)\right)+I_{k}\left(t_{k}, \varphi(0)\right) \leqslant d_{k} V\left(t_{k}^{-}, \varphi(0)\right)$ for all $\varphi \in$ $P C\left([-\tau, 0] ; \mathbb{R}^{n}\right), k \in \mathbb{Z}_{+} ;$

(iii) $D^{+} V(t, \varphi) \leqslant \eta_{1} V(t, \varphi(0))+\eta_{2} \sup _{\theta \in[-\tau, 0]} V(t+\theta, \varphi(\theta))$ for all $\varphi \in P C\left([-\tau, 0] ; \mathbb{R}^{n}\right)$ and $t \geqslant t_{0}, t \neq t_{k}, k \in \mathbb{Z}_{+}$;

(iv) $\ln d_{k} \leqslant \delta\left(t_{k}-t_{k-1}\right)$ for each $k \in \mathbb{Z}_{+}$;

(v) $\delta+\eta_{1}+\gamma \eta_{2}<0$, where $\gamma=\sup _{k \in \mathbb{Z}_{+}}\left\{e^{\delta\left(t_{k}-t_{k-1}\right)}\right.$, $\left.1 / e^{\delta\left(t_{k}-t_{k-1}\right)}\right\}$.

Then, system (21) is exponentially stable for any time delay $\tau \in(0, \infty)$, and the convergence rate should not be greater than $\lambda / p$, where $\lambda$ is the unique positive solution of $\lambda+\delta+$ $\eta_{1}+\gamma \eta_{2} e^{\lambda \tau}=0$.

In particular, if one takes $d_{k} \equiv d$ for all $k \in \mathbb{Z}_{+}$, then suppose the impulsive instances $t_{k}$ satisfy

$$
\Delta_{\text {sup }}=\sup _{k \in \mathbb{Z}_{+}}\left\{t_{k}-t_{k-1}\right\}<\infty, \quad \Delta_{\text {inf }}=\inf _{k \in \mathbb{Z}_{+}}\left\{t_{k}-t_{k-1}\right\}>0 .
$$

For system (21), Theorem 7 yields the following result.

Theorem 8. Assume that $V \in v_{0}$ and there exist constants $p>$ $0, c_{1}>0, c_{2}>0, d>0, \varrho>0, \eta_{2} \geqslant 0$, and $\eta_{1}$ such that

(i) $c_{1}|x|^{p} \leqslant V(t, x) \leqslant c_{2}|x|^{p}$ for all $(t, x) \in\left[t_{0}-\tau, \infty\right) \times \mathbb{R}^{n}$;

(ii) $V\left(t_{k}, \varphi(0)\right)+I_{k}\left(t_{k}, \varphi(0)\right) \leqslant d V\left(t_{k}^{-}, \varphi(0)\right)$ for all $\varphi \in$ $P C\left([-\tau, 0] ; \mathbb{R}^{n}\right), k \in \mathbb{Z}_{+}$;

(iii) $D^{+} V(t, \varphi) \leqslant \eta_{1} V(t, \varphi(0))+\eta_{2} \sup _{\theta \in[-\tau, 0]} V(t+\theta, \varphi(\theta))$ for all $\varphi \in P C\left([-\tau, 0] ; \mathbb{R}^{n}\right)$ and $t \geqslant t_{0}, t \neq t_{k}, k \in \mathbb{Z}_{+}$;

(iv) $\eta_{1}+\eta_{2} g(d)+\ln d / \varrho<0$, where $g(d)=d^{\Delta_{\text {sup }} / \varrho}$ if $d>1$; $g(d)=1$ if $d=1 ; g(d)=1 / d^{\Delta_{\text {inf }} / \varrho}$ if $d<1$.

Then, for any $\tau \in(0, \infty)$, when $d>1$, system (21) is exponentially stable with impulse time sequences that satisfy $\Delta_{\text {inf }} \geqslant \varrho ;$ when $d=1$, system (21) is exponentially stable with any impulse time sequences; when $d<1$, system (21) is exponentially stable with impulse time sequences that satisfy $\Delta_{\text {sup }} \leqslant$ $\varrho$.

Proof. We just need to apply Theorem 7 with $\delta=\ln d / \varrho$ and $\gamma=g(d)$

Remark 9. When $d>1$, the Lyapunov function $V$ may jump up along the state trajectories of system (21) at impulse times $t_{k}$. Thus, the impulses may be viewed as disturbances; that is, they potentially destroy the stability of continuous system. In this case, it is required that the impulses do not occur too frequently.

Remark 10. When $d<1$, the Lyapunov function $V$ may jump down along the state trajectories of system (21) at impulse times $t_{k}$. Thus, the impulses may be treated as a stabilizing factor; that is, they may be used to stabilize an unstable continuous system. In this case, the impulses must take place frequently enough, and their amplitude must be suitably related to the growth rate of $V$. 
Remark 11. When $d=1$, both the continuous dynamics and the discrete dynamics are stable, so the system can preserve exponential stability regardless of how often or how seldom impulses occur.

\section{Applications and Example}

Consider the following linear impulsive systems with timevarying delays:

$$
\begin{gathered}
\dot{x}(t)=A x(t)+B x(t-r(t)), \quad t \neq t_{k}, t \geqslant t_{0}, \\
\Delta x\left(t_{k}\right)=x\left(t_{k}\right)-x\left(t_{k}^{-}\right)=C_{k} x\left(t_{k}^{-}\right)+D_{k} x\left(t_{k}^{-}-r\left(t_{k}\right)\right), \\
k \in \mathbb{Z}_{+}, \\
x_{t_{0}}=\phi(s), \quad s \in[-\tau, 0],
\end{gathered}
$$

where $x(t) \in \mathbb{R}^{n}$ is the system state vector, $A, B, C_{k}$, and $D_{k}$ are $n \times n$ matrices, and $r: \mathbb{R}_{+} \rightarrow[0, \tau]$ with $\tau<\infty$ is the time-varying delay.

Theorem 12. Assume that there exist a matrix $P>0$ and several constants $d_{1}>0, d_{2} \geqslant 0, \eta_{2} \geqslant 0$, and $\eta_{1}$ such that

(i) the following matrix inequalities hold:

$$
\begin{gathered}
{\left[\begin{array}{ccc}
-d_{1} P & 0 & \left(I+C_{k}\right)^{T} P \\
* & -d_{2} P & D_{k}^{T} P \\
* & * & -P
\end{array}\right] \leqslant 0} \\
{\left[\begin{array}{cc}
P A+A^{T} P-\eta_{1} P & P B \\
* & -\eta_{2} P
\end{array}\right] \leqslant 0}
\end{gathered}
$$

(ii) $\ln \left(d_{1}+d_{2} \max _{\theta \in[-\tau, 0]} e^{\eta_{1} \theta}\right) \leqslant \delta\left(t_{k}-t_{k-1}\right)$;

(iii) $\delta+\eta_{1}+\eta_{2} \gamma<0$, where $\gamma=\sup _{k \in \mathbb{Z}_{+}}\left\{e^{\delta\left(t_{k}-t_{k-1}\right)}\right.$, $\left.1 / e^{\delta\left(t_{k}-t_{k-1}\right)}\right\}$.

Then, system (23) is exponentially stable, and the convergence rate should not be greater than $\lambda / 2$, where $\lambda$ is the unique positive solution of $\lambda+\delta+\eta_{1}+\gamma \eta_{2} e^{\lambda \tau}=0$.

Proof. Let $V(t, x)=x^{T} P x, I_{k}\left(t_{k}, x\left(t_{k}^{-}\right)\right)=C_{k} x\left(t_{k}^{-}\right)$, and $J_{k}\left(t_{k}, x_{t_{k}^{-}}\right)=D_{k} x\left(t_{k}^{-}-r\left(t_{k}\right)\right)$. Then, (24) combined with Schur complement yields

$$
\left[\begin{array}{cc}
-d_{1} P & 0 \\
0 & -d_{2} P
\end{array}\right]+\left[\begin{array}{ll}
I+C_{k} & D_{k}
\end{array}\right]^{T} P\left[\begin{array}{ll}
I+C_{k} & D_{k}
\end{array}\right] \leqslant 0 .
$$

Thus, for $\varphi \in P C\left([-\tau, 0] ; \mathbb{R}^{n}\right), t=t_{k}, k \in \mathbb{Z}_{+}$, using the second equation of (23), we get

$$
\begin{aligned}
& V\left(t_{k}, \varphi(0)+I_{k}\left(t_{k}, \varphi(0)\right)+J_{k}\left(t_{k}, \varphi\right)\right) \\
&=\left[\begin{array}{c}
\varphi(0) \\
\varphi\left(-r\left(t_{k}\right)\right)
\end{array}\right]^{T}\left[\begin{array}{ll}
I+C_{k} & D_{k}
\end{array}\right]^{T} P\left[\begin{array}{ll}
I+C_{k} & D_{k}
\end{array}\right] \\
& \times\left[\begin{array}{c}
\varphi(0) \\
\varphi\left(-r\left(t_{k}\right)\right)
\end{array}\right] \\
& \leqslant \\
& \quad\left[\begin{array}{c}
\varphi(0) \\
\varphi\left(-r\left(t_{k}\right)\right)
\end{array}\right]^{T}\left[\begin{array}{cc}
d_{1} P & 0 \\
0 & d_{2} P
\end{array}\right]\left[\begin{array}{c}
\varphi(0) \\
\varphi\left(-r\left(t_{k}\right)\right)
\end{array}\right] \\
& \leqslant d_{1} V\left(t_{k}^{-}, \varphi(0)\right)+d_{2} \sup _{\theta \in[-\tau, 0]} V\left(t_{k}^{-}+\theta, \varphi(\theta)\right) .
\end{aligned}
$$

In view of (25), for $\varphi \in P C\left([-\tau, 0] ; \mathbb{R}^{n}\right)$ and $t \neq t_{k}, k \in \mathbb{Z}_{+}$, we have

$$
\begin{aligned}
D^{+} V(t, \varphi) & =\left[\begin{array}{c}
\varphi(0) \\
\varphi(-r(t))
\end{array}\right]^{T}\left[\begin{array}{cc}
P A+A^{T} P & P B \\
* & 0
\end{array}\right]\left[\begin{array}{c}
\varphi(0) \\
\varphi(-r(t))
\end{array}\right] \\
& \leqslant\left[\begin{array}{c}
\varphi(0) \\
\varphi(-r(t))
\end{array}\right]^{T}\left[\begin{array}{cc}
\eta_{1} P & 0 \\
* & \eta_{2} P
\end{array}\right]\left[\begin{array}{c}
\varphi(0) \\
\varphi(-r(t))
\end{array}\right] \\
& \leqslant \eta_{1} V(t, \varphi(0))+\eta_{2} \sup _{\theta \in[-\tau, 0]} V(t+\theta, \varphi(\theta)) .
\end{aligned}
$$

Consequently, the conclusion follows from Theorem 4 immediately, and the proof is complete.

Consider the special case $D_{k} \equiv 0$; from Theorem 7 and using the similar method in the proof of Theorem 12, we can obtain the following results.

Theorem 13. Let $D_{k} \equiv 0, \Delta_{\text {sup }}=\sup _{k \in \mathbb{Z}_{+}}\left\{t_{k}-t_{k-1}\right\}<\infty$, and $\Delta_{\text {inf }}=\inf _{k \in \mathbb{Z}_{+}}\left\{t_{k}-t_{k-1}\right\}>0$. Assume that there exist a matrix $P>0$ and several constants $d>0, \varrho>0, \eta_{2} \geqslant 0$, and $\eta_{1}$ such that

(i) the following matrix inequalities hold:

$$
\begin{gathered}
\left(I+C_{k}\right)^{T} P\left(I+C_{k}\right) \leqslant d P, \\
{\left[\begin{array}{cc}
P A+A^{T} P-\eta_{1} P & P B \\
* & -\eta_{2} P
\end{array}\right] \leqslant 0}
\end{gathered}
$$

(ii) $\eta_{1}+\eta_{2} g(d)+\ln d / \varrho<0$, where $g(d)=d^{\Delta_{\text {sup }}} / \varrho$ if $d>1$; $g(d)=1$ if $d=1 ; g(d)=1 / d^{\Delta_{\text {inf }} / \varrho}$ if $d<1$.

Then, for any $\tau \in(0, \infty)$, when $d>1$, system (23) is exponentially stable with impulse time sequences that satisfy $\Delta_{\text {inf }} \geqslant \varrho$; when $d=1$, system (23) is exponentially stable with any impulse time sequences; when $d<1$, system (23) is exponentially stable with impulse time sequences that satisfy $\Delta_{\text {sup }} \leqslant \varrho$. 
Example 14. Consider the following first-order impulsive delayed neural network:

$$
\begin{gathered}
\dot{x}(t)=-a x(t)+b_{0} f(x(t))+b_{1} f(x(t-\tau)), \quad t \neq t_{k}, t \geqslant t_{0}, \\
\Delta x\left(t_{k}\right)=c x\left(t_{k}^{-}\right)+d x\left(t_{k}^{-}-\tau\right), \quad k \in \mathbb{Z}_{+},
\end{gathered}
$$

where $a=2.5, b_{0}=-1.5, b_{1}=0.5, c=0.2, d=0.1$, and $\tau=0.05 . f(x)=\tanh (x)$, and $t_{k}=\sigma k, k \in \mathbb{Z}_{+}, \sigma>0$, is a constant. It is easy to see that system without impulses is exponentially stable and the impulses are destabilizing since $c, d>0$. Choose $V(t, x)=x^{2} / 2$; then we obtain that conditions (i) and (ii) of Theorem 4 hold with $c_{1}=c_{2}=1 / 2$, $p=2, d_{1 k}=(1+c)(1+c+d)=1.56$, and $d_{2 k}=d(1+c+d)=$ 0.13 .

For $t \neq t_{k}$, by simple calculation, we have

$$
\begin{aligned}
D^{+} V(t, x(t))= & -a x^{2}(t)+b_{0} x(t) f(x(t)) \\
& +b_{1} x(t) f(x(t-\tau)) \\
\leqslant & -\left(a+b_{0}-\frac{1}{2} b_{1}\right) x^{2}(t)+\frac{1}{2} b_{1} x^{2}(t-\tau) \\
= & -\left(2 a+2 b_{0}-b_{1}\right) V(t, x(t)) \\
& +b_{1} V(t, x(t-\tau)),
\end{aligned}
$$

which implies that condition (iii) of Theorem 4 holds with $\eta_{1}=-\left(2 a+2 b_{0}-b_{1}\right)=-1.5$ and $\eta_{2}=b_{1}=0.5$. Note that $t_{k}=\sigma k$, so we can take $\delta=\ln \left(d_{1 k}+d_{2 k} e^{-\eta_{1} \tau}\right) /\left(t_{k}-t_{k-1}\right)=$ $\ln \left(1.56+0.13 e^{0.075}\right) / \sigma$ and $\gamma=d_{1 k}+d_{2 k} e^{-\eta_{1} \tau}=1.56+$ $0.13 e^{0.075}$. Then, by Theorem 4 , system (30) is exponentially stable over any impulse time sequences satisfying $t_{k}-t_{k-1} \geqslant$ $\sigma>\ln \left(1.56+0.13 e^{0.075}\right) /\left[1.5-0.5\left(1.56+0.13 e^{0.075}\right)\right]=0.4298$.

Remark 15. Since the system without impulses is exponentially stable and the impulses are destabilizing, the existing results in $[29,32]$ cannot be applied to $(30)$. The Razumikhintype theorem in [31] is also not convenient to be applied to this system since it is not easy to find an appropriate constant $q>1$ to satisfy the Razumikhin-type condition.

Example 16. Consider system (23) with the following parameters:

$$
\begin{array}{cc}
A=\left[\begin{array}{cc}
2 & 1 \\
0.8 & 2
\end{array}\right], & B=\left[\begin{array}{cc}
1 & -0.2 \\
0.3 & 1
\end{array}\right], \\
C_{k} \equiv\left[\begin{array}{cc}
-0.3 & 0 \\
0 & -0.3
\end{array}\right], & D_{k} \equiv\left[\begin{array}{cc}
0.2 & 0 \\
0 & 0.2
\end{array}\right], \quad k \in \mathbb{Z}_{+},
\end{array}
$$

and $r(t)=8+0.02[\sin (t)]$, where [.] denotes the integer function. Let $\tau=8.2, d_{1}=0.49, d_{2}=0.04, \eta_{1}=7, \eta_{2}=1$, $\gamma=0.53, \delta=-7.9360$, and $t_{k}-t_{k-1} \leqslant 0.08$. It is easy to verify that

$$
\begin{gathered}
\eta_{1}+\eta_{2} \gamma+\delta=-0.4060<0, \\
\ln \left(d_{1}+d_{2} \max _{\theta \in[-\tau, 0]} e^{\eta_{1} \theta}\right) \\
=\ln 0.53=-0.6349<-7.9360 \times 0.08 \leqslant \delta\left(t_{k}-t_{k-1}\right) .
\end{gathered}
$$

Solving the linear matrix inequalities (24) and (25) in Theorem 12, we obtain the following feasible solution $P=$ $\left[\begin{array}{ll}0.3507 & 0.0332 \\ 0.0332 & 0.3076\end{array}\right]$. Then, by Theorem 12 , we know the given system is exponentially stable.

Remark 17. In Example 16, the impulses are used to stabilize an unstable system. In this case, the impulses must be frequent enough, and their amplitude must be suitably related to the growth rate of the continuous flow.

\section{Conclusions}

This paper has studied the exponential stability of impulsive delay systems in which the state variables on the impulses are related to the time delay. By using the Lyapunov function method, some criteria on the exponential stability are established. Moreover, the stability criteria obtained are applied to linear impulsive systems with time-varying delays, and a set of sufficient conditions for exponential stability is provided in terms of matrix inequalities. The obtained results improve and complement some recent works. Two examples have been given to illustrate the effectiveness and advantages of the results obtained.

\section{Conflict of Interests}

The authors declare that there is no conflict of interests regarding the publication of this paper.

\section{Acknowledgments}

This work was supported by the National Natural Science Foundation of China (61273126, 11226247, and 11301004), the Anhui Provincial Nature Science Foundation (1308085QA15, 1308085MA01), the Key Natural Science Foundation (KJ2009A49), the Foundation of Anhui Education Bureau (KJ2012A019), and the 211 Project of Anhui University (32030018 and 33010205).

\section{References}

[1] V. Lakshmikantham, D. D. Baĭnov, and P. S. Simeonov, Theory of Impulsive Differential Equations, vol. 6 of Series in Modern Applied Mathematics, World Scientific, Singapore, 1989.

[2] K. Gopalsamy and B. G. Zhang, "On delay differential equations with impulses," Journal of Mathematical Analysis and Applications, vol. 139, no. 1, pp. 110-122, 1989. 
[3] G. Ballinger and X. Liu, "Existence, uniqueness and boundedness results for impulsive delay differential equations," Applicable Analysis, vol. 74, no. 1-2, pp. 71-93, 2000.

[4] W. M. Haddad, V. Chellaboina, and S. G. Nersesov, Impulsive and Hybrid Dynamical Systems: Stability, Dissipativity, and Control, Princeton Series in Applied Mathematics, Princeton University Press, Princeton, NJ, USA, 2006.

[5] W. Chen and W. Zheng, "On improved robust stabilization of uncertain systems with unknown input delay," Automatica, vol. 42, no. 6, pp. 1067-1072, 2006.

[6] E. Fridman and U. Shaked, "An improved stabilization method for linear time-delay systems," IEEE Transactions on Automatic Control, vol. 47, no. 11, pp. 1931-1937, 2002.

[7] S. Xu and T. Chen, "Robust $H_{\infty}$ control for uncertain stochastic systems with state delay," IEEE Transactions on Automatic Control, vol. 47, no. 12, pp. 2089-2094, 2002.

[8] D. Yue, "Robust stabilization of uncertain systems with unknown input delay," Automatica, vol. 40, no. 2, pp. 331-336, 2004.

[9] L. Huang and X. Mao, "Robust delayed-state-feedback stabilization of uncertain stochastic systems," Automatica, vol. 45, no. 5, pp. 1332-1339, 2009.

[10] D. Wang, P. Shi, J. Wang, and W. Wang, "Delay-dependent exponential $H_{\infty}$ filtering for discrete-time switched delay systems," International Journal of Robust and Nonlinear Control, vol. 22, no. 13, pp. 1522-1536, 2012.

[11] W. Chen and W. Zheng, "Robust stability and $H_{\infty}$-control of uncertain impulsive systems with time-delay," Automatica, vol. 45, no. 1, pp. 109-117, 2009.

[12] G. Zong, S. Xu, and Y. Wu, "Robust $H_{\infty}$ stabilization for uncertain switched impulsive control systems with state delay: an LMI approach," Nonlinear Analysis: Hybrid Systems, vol. 2, no. 4, pp. 1287-1300, 2008.

[13] B. Liu, K. L. Teo, and X. Liu, "Robust exponential stabilization for large-scale uncertain impulsive systems with coupling timedelays," Nonlinear Analysis: Theory, Methods \& Applications, vol. 68, no. 5, pp. 1169-1183, 2008.

[14] X. Liu, S. Zhong, and X. Ding, "Robust exponential stability of impulsive switched systems with switching delays: a Razumikhin approach," Communications in Nonlinear Science and Numerical Simulation, vol. 17, no. 4, pp. 1805-1812, 2012.

[15] A. Anokhin, L. Berezansky, and E. Braverman, "Exponential stability of linear delay impulsive differential equations," Journal of Mathematical Analysis and Applications, vol. 193, no. 3, pp. 923-941, 1995.

[16] Z. Luo and J. Shen, "Stability and boundedness for impulsive functional differential equations with infinite delays," Nonlinear Analysis: Theory, Methods \& Applications, vol. 46, no. 4, pp. 475493, 2001.

[17] I. M. Stamova and G. T. Stamov, "Lyapunov-Razumikhin method for impulsive functional differential equations and applications to the population dynamics," Journal of Computational and Applied Mathematics, vol. 130, no. 1-2, pp. 163-171, 2001.

[18] Z. Luo and J. Shen, "Impulsive stabilization of functional differential equations with infinite delays," Applied Mathematics Letters, vol. 16, no. 5, pp. 695-701, 2003.

[19] Y. Zhang and J. Sun, "Stability of impulsive infinite delay differential equations," Applied Mathematics Letters, vol. 19, no. 10, pp. 1100-1106, 2006.
[20] Z. Chen and X. Fu, "New Razumikhin-type theorems on the stability for impulsive functional differential systems," Nonlinear Analysis: Theory, Methods \& Applications, vol. 66, no. 9, pp. 2040-2052, 2007.

[21] A. Weng and J. Sun, "Impulsive stabilization of second-order delay differential equations," Nonlinear Analysis: Real World Applications, vol. 8, no. 5, pp. 1410-1420, 2007.

[22] X. Fu and X. Li, "Razumikhin-type theorems on exponential stability of impulsive infinite delay differential systems," Journal of Computational and Applied Mathematics, vol. 224, no. 1, pp. $1-10,2009$.

[23] Z. Luo and J. Shen, "Stability of impulsive functional differential equations via the Liapunov functional," Applied Mathematics Letters, vol. 22, no. 2, pp. 163-169, 2009.

[24] S. Peng and L. Yang, "Global exponential stability of impulsive functional differential equations via Razumikhin technique," Abstract and Applied Analysis, vol. 2010, Article ID 987372, 11 pages, 2010.

[25] X. Li, "Uniform asymptotic stability and global stabiliy of impulsive infinite delay differential equations," Nonlinear Analysis: Theory, Methods \& Applications, vol. 70, no. 5, pp. 1975-1983, 2009.

[26] X. Li, "New results on global exponential stabilization of impulsive functional differential equations with infinite delays or finite delays," Nonlinear Analysis: Real World Applications, vol. 11, no. 5, pp. 4194-4201, 2010.

[27] F. Lian, J. Moyne, and D. Tilbury, "Modelling and optimal controller design of networked control systems with multiple delays," International Journal of Control, vol. 76, no. 6, pp. 591606, 2003.

[28] A. Khadra, X. Liu, and X. Shen, "Analyzing the robustness of impulsive synchronization coupled by linear delayed impulses," IEEE Transactions on Automatic Control, vol. 54, no. 4, pp. 923928, 2009.

[29] Y. Zhang and J. Sun, "Stability of impulsive functional differential equations," Nonlinear Analysis: Theory, Methods \& Applications, vol. 68, no. 12, pp. 3665-3678, 2008.

[30] W. Chen and W. Zheng, "Exponential stability of nonlinear time-delay systems with delayed impulse effects," Automatica, vol. 47, no. 5, pp. 1075-1083, 2011.

[31] P. Cheng, Z. Wu, and L. Wang, "New results on global exponential stability of impulsive functional differential systems with delayed impulses," Abstract and Applied Analysis, vol. 2012, Article ID 376464, 13 pages, 2012.

[32] D. Lin, X. Li, and D. O’Regan, "Stability analysis of generalized impulsive functional differential equations," Mathematical and Computer Modelling, vol. 55, no. 5-6, pp. 1682-1690, 2012.

[33] F. Yao, F. Deng, and P. Cheng, "Exponential stability of impulsive stochastic functional differential systems with delayed impulses," Abstract and Applied Analysis, vol. 2013, Article ID 548712, 8 pages, 2013.

[34] X. Liu and G. Ballinger, "Uniform asymptotic stability of impulsive delay differential equations," Computers \& Mathematics with Applications, vol. 41, no. 7-8, pp. 903-915, 2001.

[35] Q. Wang and X. Liu, "Exponential stability for impulsive delay differential equations by Razumikhin method," Journal of Mathematical Analysis and Applications, vol. 309, no. 2, pp. 462-473, 2005. 


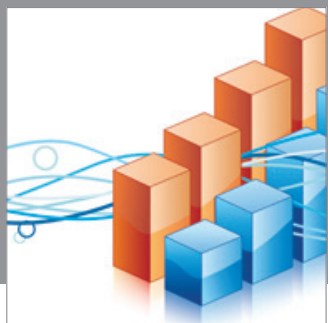

Advances in

Operations Research

mansans

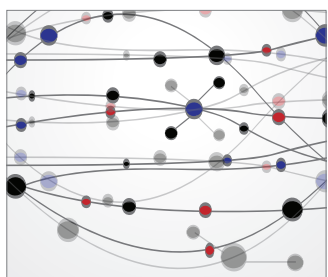

The Scientific World Journal
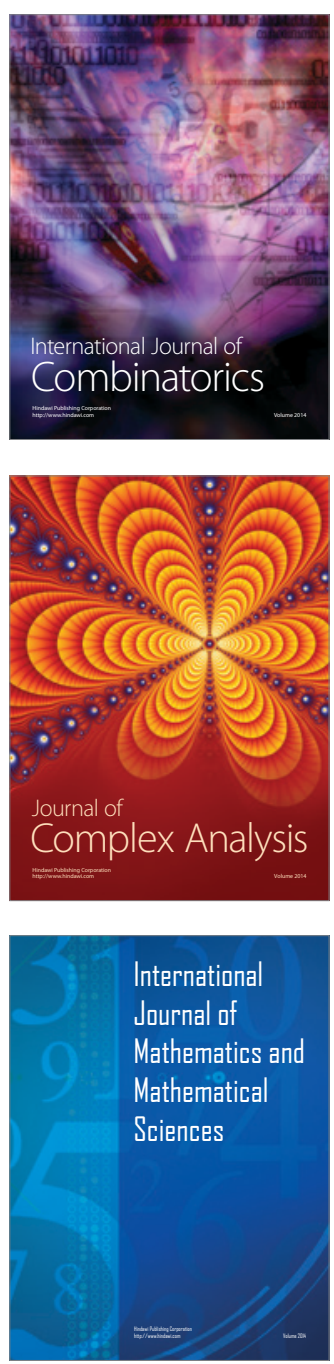
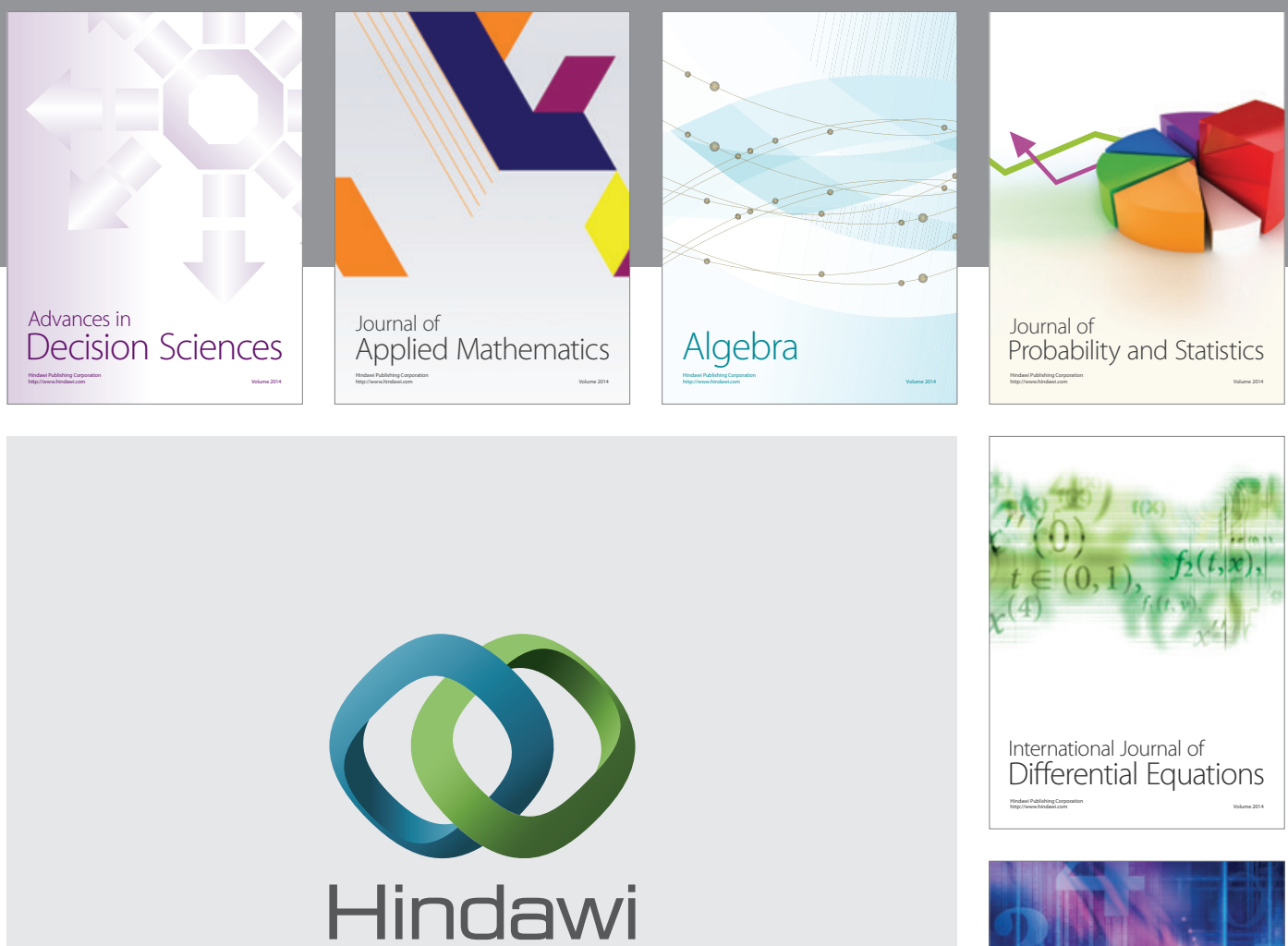

Submit your manuscripts at http://www.hindawi.com
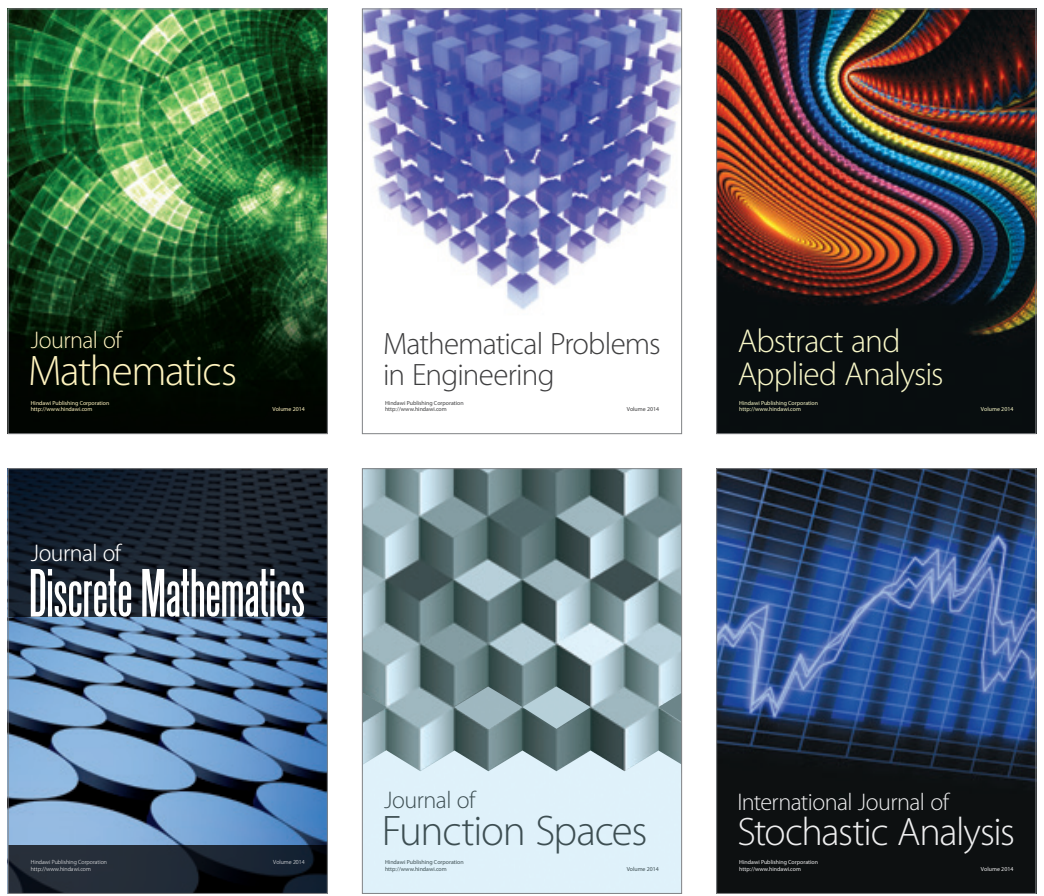

Journal of

Function Spaces

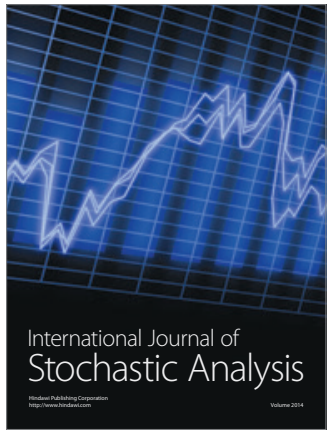

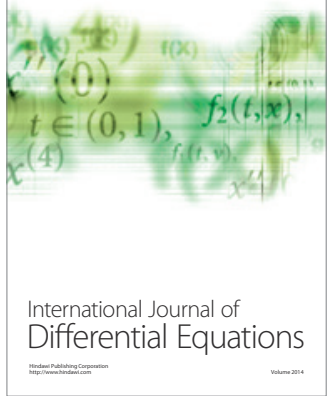
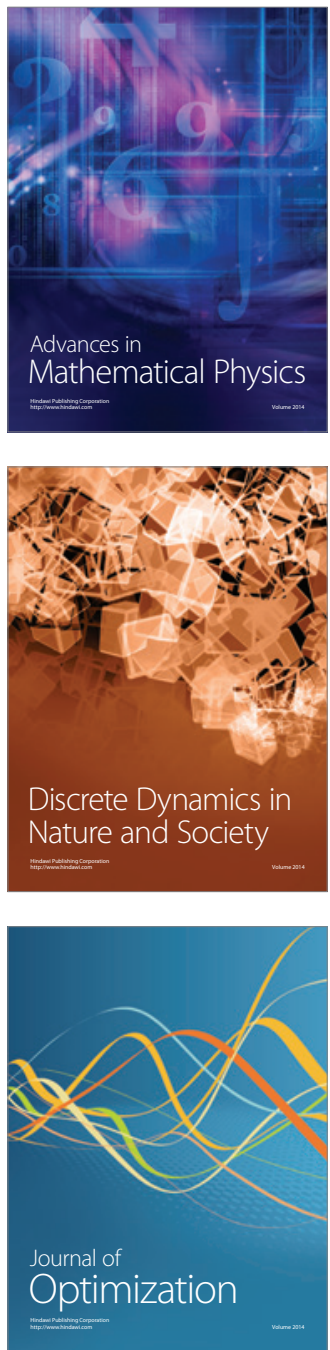